\title{
Rehabilitación estética y funcional en dientes con atrición y restablecimiento de dimensión vertical por pérdida de soporte oclusal posterior: Reporte de caso
}

\author{
Aesthetic and functional rehabilitation in teeth with attrition and restoration of vertical \\ dimension due to loss of posterior occlusal support: Case report \\ Martha Acosta Molina ${ }^{1 a}$, Osmani Guevara Cabrera ${ }^{2 a}$ Ana Armas Vega ${ }^{3 b}$, Esteban Vizcaíno ${ }^{1 \mathrm{c}}$, Alexander Cruz Gallegos ${ }^{3 a}$
}

\section{RESUMEN}

La rehabilitación estética y funcional es considerada uno de los maximos objetivos de cualquier tratamiento odontológico, con una base científica sustentada y actualizada, buscando siempre un diagnóstico completo e integral, y un plan de tratamiento acorde a las necesidades del paciente, con un abordaje multidisciplinario basado en el diálogo constante entre el profesional, el paciente y el técnico dental. El objetivo de este reporte, es describir paso a paso las etapas clínicas de un tratamiento multidisciplinario el cual se base en el diagnóstico previo y la planificación; buscando la interacción de diferentes especialidades: periodoncia, rehabilitación oral, odontología estética, oclusión, y un trabajo integral con el laboratorio dental, para lograr la estabilidad de los tejidos periodontales y dentales. Recuperar la función masticatoria y obtener la satisfacción estética y funcional del paciente. Paciente femenino de 54 años de edad, sistémicamente comprometida, con patologías previas, con ausencia de dientes, espacios edentulo largos, atricción dental, perdida dimensión vertical y alteraciones funcionales y estéticas. Con un régimen estricto de los protocolos establecidos, se cumplió lo planificado. El éxito del tratamiento se fundamentó en: 1) mantener la estabilidad de los tejidos periodontales y óseos dentales. 2) recuperación de la función masticatoria y devolver la dimensión vertical; 3) obtención de un resultado estético aceptable; 4) satisfacción estética y funcional de la paciente.

Palabras Clave: Rehabilitación protésica; Estética dental; Dimensión vertical; Oclusión dental. (Fuente: DeCS BIREME)

\begin{abstract}
A esthetic and functional rehabilitation is considered the maximum objective of any dental treatment, with a sustained and updated scientific base, always seeking a complete and comprehensive diagnosis, focused on presenting a treatment plan according to comprehensive needs of the patient, executed in a conscious way with an established multidisciplinary approach, with foundations based on constant dialogue between the professional, the patient and the dental technician. The objective of this report is to describe step by step the clinical stages of a multidisciplinary treatment which is based on prior diagnosis and planning; looking for the interaction of different specialties: periodontics, oral rehabilitation, aesthetic dentistry, occlusion, and an integral work with the dental laboratory, to achieve the stability of periodontal and dental tissues. Thus recovering the masticatory function and obtaining an acceptable aesthetic result to achieve the aesthetic and functional satisfaction that the patient sought. A 54-year-old female patient, systemically compromised, with previous pathologies, with the absence of teeth, long edentulous spaces, dental attrition, loss of vertical dimension and functional and aesthetic alterations. With a strict regime of established protocols, what was planned was fulfilled. The success of the treatment was based on: 1) maintaining the stability of dental periodontal and bone tissues. 2) recovery of masticatory function and return the vertical dimension; 3) obtaining an acceptable aesthetic result; 4) aesthetic and functional satisfaction of the patient.
\end{abstract}

Key words: Prosthetic rehabilitation; Esthetics; Dental; vertical dimension; Oclusión Dental. (Source: MeSH NLM)

${ }^{1}$ Universidad Tecnológica de Ecuador (UTE). Ecuador

2 Universidad Central del Ecuador. Ecuador

${ }^{3}$ Universidad Tecnológica Equinoccial del Ecuador

${ }^{\text {a }}$ Odontólogo

PhD en operatoria dental, docente

${ }^{c}$ Rehabilitador oral

\section{Correspondencia:}

Alex Cruz Gallegos

Dirección: De los nardos 5862 y Leonardo Murialdo. Ecuador

Correo electrónico: alexandercruz1993@hotmail.com

Citar como: Acosta Molina M, Guevara Cabrera O, Armas Vega A, Vizcaino E, Cruz Gallegos A. Rehabilitación estética y funcional en dientes con atrición y restablecimiento de dimensión vertical por pérdida de soporte oclusal posterior: Reporte de caso. KIRU. 2020;17(3):149-156. https://doi.org/10.24265/kiru.2020.v17n3.06 


\section{INTRODUCCIÓN}

Una de las manifestaciones más comunes de esta inestabilidad oclusal, detectada, constituye la pérdida de tejido dentario a nivel de bordes incisales, denominada atrición cuya manifestación principal constituye el desgaste dental causado por el contacto interdental, desencadenante de facetas de desgaste en la superficie del esmalte que se evidencia con mayor frecuencia en los bordes incisales de los dientes anteriores y oclusales de los dientes posteriores, detectados tras una evaluación minuciosamente ${ }^{(1-3)}$.

Este desgaste a nivel dental esta asociado en gran medida con la perdida de contención posterior, producida por la perdida de dientes posteriores, premolares y molares desencadenando una perdida de estabilidad mandibular durante el cierre dental por la perdida de dispersion de las fuerzas oclusales $^{(4,5)}$

Al ser los dientes posteriores, los elementos decisivos de soporte de las fuerzas oclusales, la selección de los materiales a ser empleados para remplazar o restaurar sus ausencias totales o parciales, se convierte en uno de los elementos fundamentales del éxito en todo tratamiento restaurador ${ }^{(6)}$, tanto pesando en la funcion como en la estética, buscando conseguir una oclusión funcional óptima, con contactos uniformes y simultáneos a nivel dental que aseguren el adecuado balance a nivel de la articulación temporo mandibular (ATM), con cóndilos mandibulares en su posición anterosuperior máxima dentro de la cavidad articular y discos articulares interpuestos entre ellos ${ }^{(7)}$.

El objetivo es describir paso a paso las etapas clínicas de un tratamiento multidisciplinario el cual se base en el diagnóstico previo y la planificación; buscando la interacción de diferentes especialidades: periodoncia, rehabilitación oral, odontología estética, oclusión, y un trabajo integral con el laboratorio dental, para lograr la estabilidad de los tejidos periodontales y dentales. En un paciente de sexo femenino, con ausencia de piezas dental, con inestabilidad oclusal y perdida de la dimensión vertical producto de la atrición; buscado devolver la estabilidad funcional de la cavidad oral, y mejorar la estética del paciente.

\section{REPORTE DE CASO}

Paciente de género femenino, de 54 años de edad, acude a la clínica de especialidades odontológicas de la Universidad Internacional del Ecuador (UIDE)
Quito-Ecuador, relatando antecedentes de hidrocefalia y mielo meningocele controlados durante su crecimiento, osteomielitis en el pie derecho y cuadros de depresión diagnosticada y en tratamiento, para un chequeo habitual. Tras firma del consentimiento informado autorizando su análisis y posterior difusión en relato de caso clínico, respetando los principios de ética. Un dialogo y análisis inicial permitió conocer que 20 años atrás, la paciente inicio un proceso paulatino de perdida dental en el sector posterior y más recientemente a nivel anterior, lo que concluyo en la pérdida de confianza de la paciente al sonreír.

Al examen extraoral, la paciente no presenta ninguna patología aparente, sin embargo al trazar las líneas horizontales y verticales se observa que la paciente presenta asimetría no marcada sin deformidades a nivel facial, ausencia de ganglios inflamados a la palpación, ruidos o dolor al examen de ATM. Al examen intraoral se observa ausencia de patología aparente, evidenciándose elementos dentarios perdidos en las dos arcadas y perdida de estructura dental a nivel del borde incisal de los dientes anteriores superiores, asociada con una atricción dental, provocando una desarmonía evidente también a nivel de los tejidos gingivales, influenciando de forma negativa en la estética de la sonrisa de la paciente. Figuras 1 y 2 .

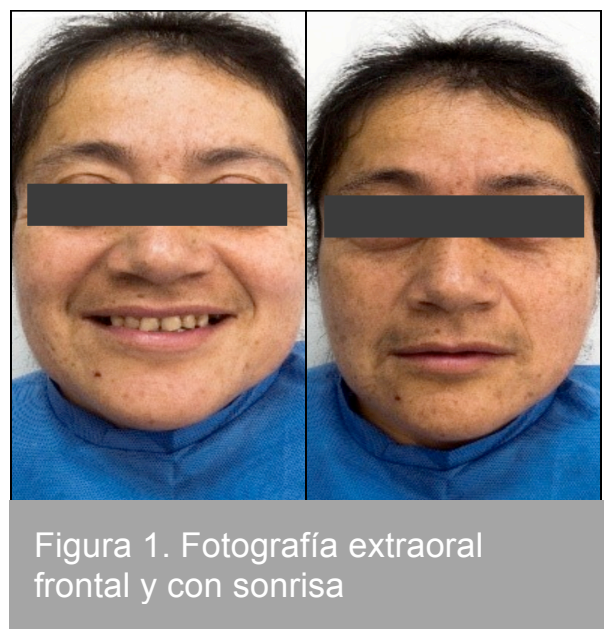

Al analizar la proporción corono raíz de todos los dientes presentes en boca, se observa que está en relación 1 a 1, considerada mínima dentro de los procesos rehabilitadores. Por otro lado, los modelos de estudio, permiten reproducir las estructuras dentales del paciente para ejecutar alternativas de planes de tratamiento, y observar Isu relación oclusal dándonos una idea de qué tipos de prótesis serían las más adecuadas ${ }^{(8)}$. 


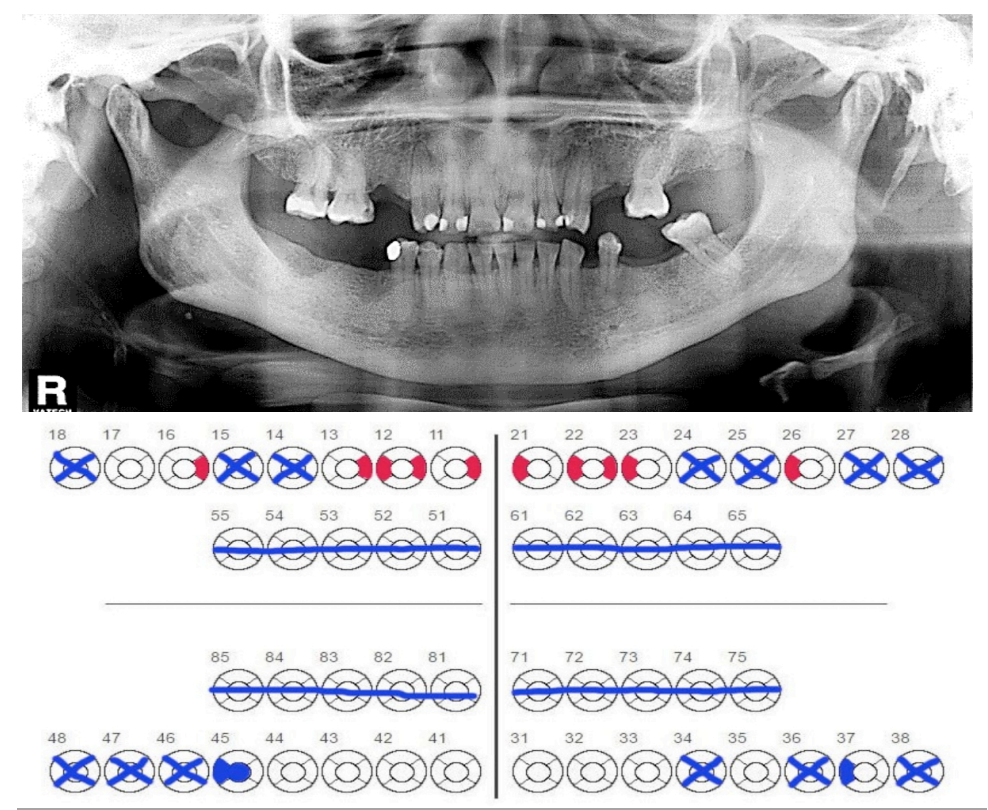

Figura 2. Radiografia panorámica y Odontograma

Fueron ejecutadas diferentes sesiones de remoción de biofilm y cálculo supra gingival, disminuyendo con ello la carga bacteriana de la cavidad bucal. Acompañado de motivación y fisioterapia oral al paciente, con la enseñanza, adiestramiento y acompañamiento a la paciente en técnicas de cepillado y manejo correcto de hilo dental. En el estudio de la relación intermaxilar, empleando un articulador semiajustable en conjunto con el arco facial fue ejecutado ${ }^{(9)}$. Mediante la elaboración del encerado diagnóstico, nos permitió constatar la necesidad de realizar una gingivectomía en el sector anterior. Figura 3.

Los análisis previos determinaron la necesidad de colocar coronas unitarias en cerámica pura en los dientes $1.1,1.2,2.1$ y 2.2 buscando maximizar la estética en la paciente. Colocar un puente de cuatro dientes en el cuadrante 1, tomando como pilares a los dientes 1.3 y 1.6 considerando como pónticos los dientes 1.4 y 1.5. De la misma manera en el cuadrante 2, fue planificada una prótesis, considerando como pilares a los dientes 2.3 y 2.6 reemplazando a 2.4 y 2.5 . Empleándose metalcerámica como material protésico, por considerarlo idóneo, pensando en las fuerzas masticatorias a soportar. Con respecto al diente 1.7 el análisis realizado permitió verificar la necesidad de ser tallado para recibir una corona metal porcelana y obtener una oclusión balanceada. El tallado inició con la preparación de guías para el tallado en la superficie vestibular de todos los dientes que recibirán prótesis fija, empleando fresas diamantadas tronco cónicas de punta redonda, calibradas al espesor necesario, considerando el material a ser empleado posteriormente.
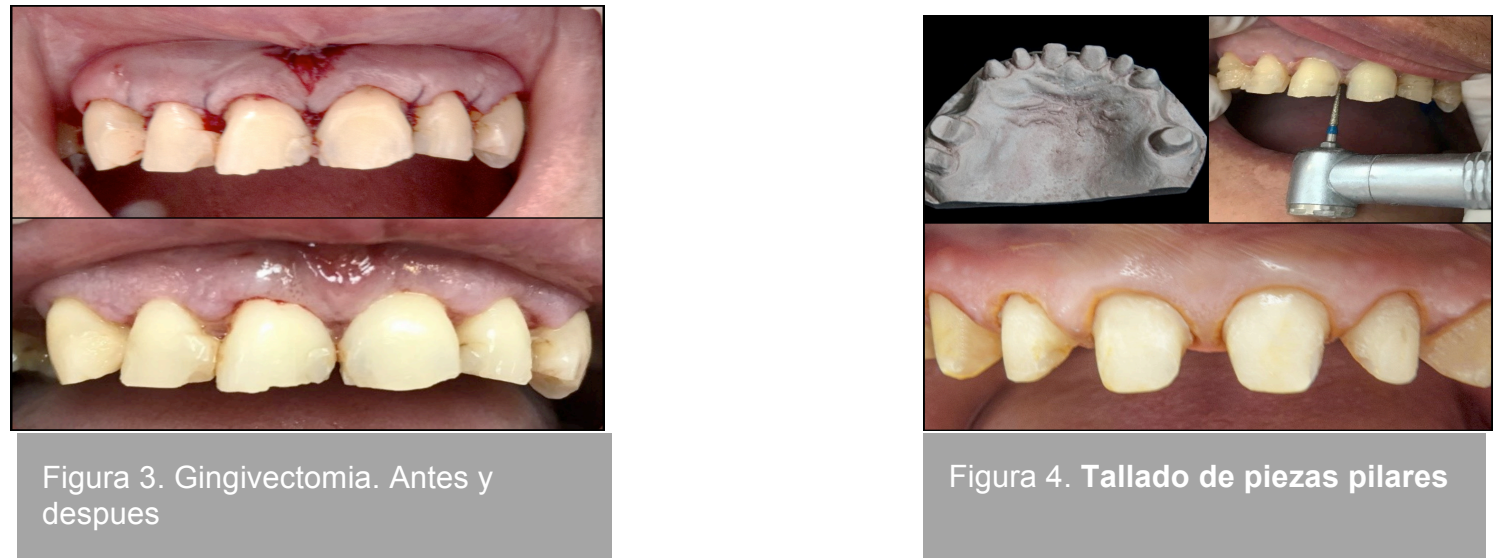
Con una profundidad de $1 \mathrm{~mm}$ para coronas de porcelana pura y de mínimo, $1.5 \mathrm{~mm}$ para las coronas de metal cerámica ${ }^{(10)}$. Se continuó con una fresa diamantada de forma troncocónico de punta redonda a la regularización de la superficie vestibular de cada diente, siempre teniendo en cuenta la salud pulpar 11. Se procedió con la eliminación de los puntos de contacto entre diente y diente, mediante una fresa diamantada con formato en fisura. El tallado prosiguió en las superficies palatinas de los dientes, respetando las vertientes que estas superficies presentan y la espesura establecida según el material. De la misma forma el tallado fue ejecutado en las superficies oclusales de los dientes posteriores, realizado proporcionando espacio suficiente en las fosas centrales, que permitan proporcionar una morfología funcional a los elementos protésicos que se pretenden ejecutar ${ }^{(11)}$.

El margen gingival de las preparaciones fue ubicado yuxtagingival, cumpliendo con las exigencias estéticas y de mantenimiento de la salud periodontal, y verificando la regularidad en

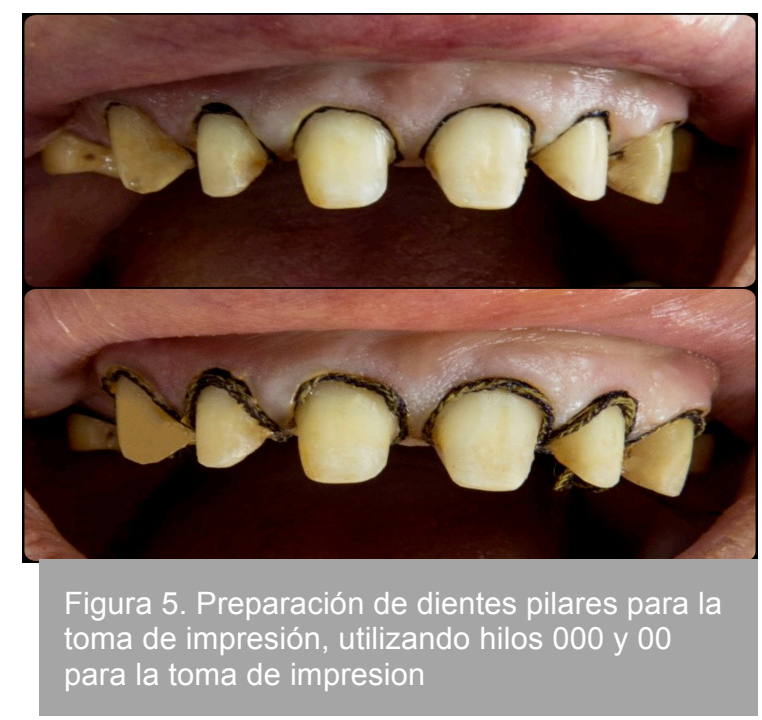

Como paso a seguir, siliconas de adición pesada y liviana, fueron dispensadas cuidando respetar su dosificación, elegidas por su buena reproducción de los detalles y excelente estabilidad dimensional evidenciada en una mayor resistencia a deformidades, manteniendo su integridad por más tiempo.

Se procede a tomar el registro de mordida en relación céntrica que permitirá el montaje del modelo inferior y a cementar las estructuras protésicas provisionales empleando materiales a base de hidróxido de calcio. estos márgenes, para evitar el acumulo de placa bacteriana y filtraciones de los futuros elementos protésicos. Figura 4.

La ejecución de los provisionales constituye uno de los procedimientos más esperados por el paciente y por el profesional, en el presente caso clínico debido a las expectativas estéticas mantenidas por la paciente y los objetivos funcionales buscados, estos provisionales fueron realizados con acrílico termopolimerizable, lo que nos permitió obtener una mayor adaptación al margen gingival del tallado, mejor estética $y$ cumplimiento de las funciones del sistema masticatorio. El registro del tallado definitivo, fue realizado 8 días luego del tallado dentario, con el propósito de evitar daños a los tejidos blandos inflamados o lesionados durante su preparación. Fueron insertados hilos retractores 000 seguido por hilo 00, a nivel del surco gingival, buscando obtener una separación del margen gingival con respecto al hombro de la preparación, facilitando su visibilidad. Figura 5.

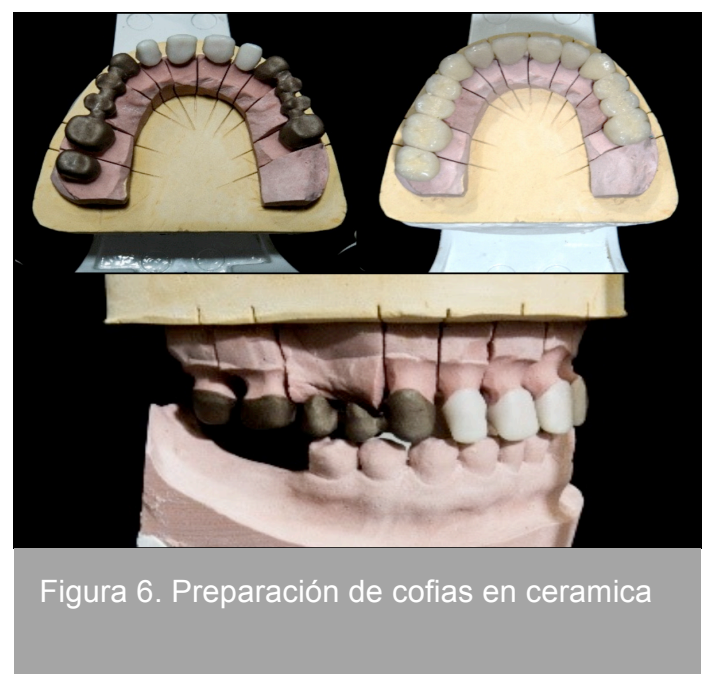

Sobre los modelos funcionales articulados, el laboratorio dental procede a elaborar siguiendo los protocolos propios exigidos por los fabricantes de cada material, cofias en cerámica. Se registro de color dental, empleando escalas de color y considerando como guía los dientes inferiores presentes en boca, el cual se decidió 2A/130, según el colorímetro Ivoclar "chromascop". Figura 6. Como paso protésico final, una vez en el laboratorio, los elementos protésicos fueron revestidos por el "glasé", quedando terminado el proceso. Finalmente la protesis fue cementada. Figura 7. 
En el presente caso clínico, se decidió colocar una prótesis parcial removible en el sector inferior, por la necesidad de restituir la función al sistema masticatorio y armonizar la oclusión de la paciente. Considerando las implicancias dentales,

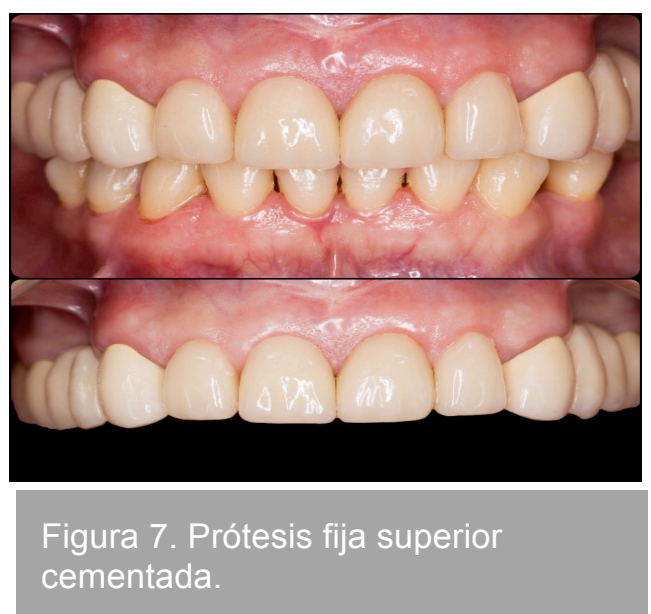

Después de la colocación de las prótesis fija y removible, se realizó el control respectivo a las 24 horas, para comprobar la estabilidad de las prótesis y aliviar todas las molestias que el paciente presentaba. En el cual se realizó un control oclusal de la prótesis inferior para controla las molestas del paciente. De esta misma manera a los 5 días el paciente acudió a la consulta, presentando molestias mínimas en el sector inferior, las cuales fueron resueltas de inmediato. relacionadas con ausencias parciales que indican que nos encontramos ante un paciente que presenta una clase de Kennedy II modificación 2, se decidió elaborar una prótesis funcional estética y confortable. Figura 8

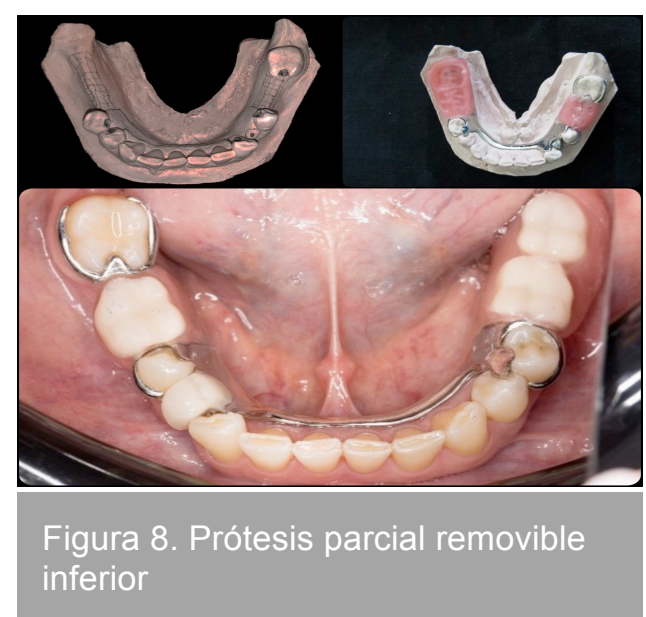

El paciente tendrá que acudir al mes, 2 meses, 4 meses, 6 meses y al año para control tanto clínico como radiográfico de las coronas de metal porcelana y porcelana pura y de la prótesis removible inferior. Para controlar la filtración y reabsorción ósea de las piezas pilas tanto en los dos tipos de prótesis. La paciente mejoró su autoestima porque se sentía segura de sonreír sin inconvenientes y estaba muy conforme con el cambio. Figura 9

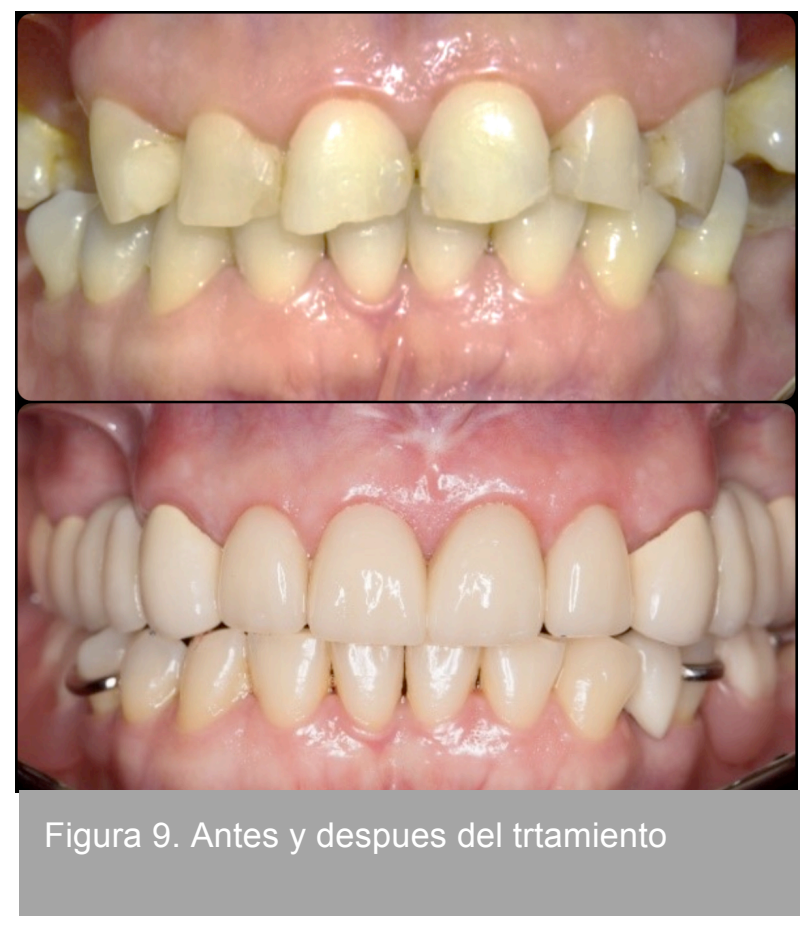




\section{DISCUSIÓN}

La pérdida dental independiente de la causa, constituye un elemento desencadenante de múltiples alteraciones a nivel oclusal, la existencia desbalance ${ }^{(12)}$. En el caso clínico relatado, el diagnóstico adecuado y un trabajo multidisciplinario donde diferentes especialistas intervinieron conjugado con una comunicación con el técnico en prótesis dental de forma constante, fueron los elementos que permitieron alcanzar el éxito reportado no solo estético tanto como funcional ${ }^{(13)}$.

La búsqueda de una sonrisa armónica en el paciente constituye un elemento decisivo y motivador cuando se trata de contar con la colaboración por parte del paciente, sin embargo la sonrisa depende de diferentes elementos, pudiendo hablarse de una estética blanca y otra rosada; en el presente caso a la paciente se le fueron expuestas las diferentes alternativas de tratamiento, considerando siempre sus necesidades, explicando las razones por las que su sonrisa fue perdida, el conocimiento y educación que la paciente contó en cada procedimiento a ser realizado consideramos permitirá cumplir a largo plazo con el éxito en los procedimientos ejecutados ${ }^{(14)}$.

El tratamiento de estos desgastes en los bordes incisales de los dientes anteriores y oclusales de los dientes postriores, requiere evaluación minuciosamente por la posible asociación con diferentes hábitos nocivos comúnmente relacionados con el desempeño laboral de quien lo padece, en el caso relatado, la atrición dental mostró asociación con la perdida de contención posterior, producida por la perdida de dientes posteriores, premolares y molares, son los dientes que dirigen la mandibula durante el cierre y resisten fuerzas oclusales, para su correcta dispersion ${ }^{(15-16)}$.

El análisis clínico y radiográfico ejecutado conjugado con el encerado permitió determinar la necesidad de un abordaje periodontal, el resultado de las acciones ejecutadas por este especialista mostró los indudables beneficios que un trabajo multidisciplinario produce. Una sonrisa armoniosa es sinónimo de belleza y juventud pero existen muchos elementos inmiscuidos en perderla y en restituirla, los tejidos gingivales constituyen el marco de la sonrisa y su restitución en el caso relatado permitió a la paciente mejorar su sonrisa y al odontólogo contar con tejido suficiente que permita un trabajo protésico respetando proporciones y medida ${ }^{(16)}$. de atricción dental que fueron el elemento que preocupó a la paciente motivándola a acudir a la clínica odontológica de la UIDE, constituye una de las manifestaciones mas evidentes de este

Una rehabilitación oral debe ser realizada para restablecer la estética dental perdida y sobre todo para recuperar la correcta oclusion, que en muchas de las veces esta pasa desapersibida por el paciente al no producir sintomas (16). En ese contexto, es el odontólogo quien tiene la obligación de diagnosticar y educar al paciente, en el caso clinico relatado la oclusión ideal fue recuperada junto con la estética del paciente; sin embargo, muchos dientes en este proceso tuvieron que ser modificados, para recibir protesis fija o protesis removible, el costo, malestar y tiempo que estas acciones merecieron constituyen muchas veces en limitantes, importantes cuando se trata de personas con medios economicos bajos, que impiden alcanzar el éxito.

El pronóstico según lo relatado se muestra favorable, los procedimientos protésicos fueron ejecutados respetando la estructura dental pero siguiendo los principios establecidos tanto para la prótesis fija como la removible ${ }^{(16)}$, sin embargo, constituyen elementos protésicos extraños construidos con materiales que intentan simular las características dentales, pero que son susceptibles de presentar cambios muchos de ellos irreversibles, dependientes de las condiciones de higiene y nutrición que el paciente realice.

La paciente del caso relatado, al perder sus dientes posteriores, modificó su masticacion, provocando que los dientes anteriores reciban cargas oclusales que no les corresponden, ocacionando este desgaste dental, alterando su estética y oclusión. Rehabilitar un paciente con patología oclusal, exige recontruciones extensas de todas las superficies dentales palatinas $y$ oclusales que presenten cambios, por medio de protesis fijas, removibles e incluso implantes ${ }^{(16)}$. En el presente caso una combinación de protesis fija en el maxilar superior y removible en la inferior fue la respuesta mas acertada, la colocación de implantes lastimosamente requeria la reconstrucción y reposición de tejidos con protesis lo cual elevaria a un mas el costo del tratamiento

Es basico entender que pese a una tendencia conservadora, el implante no siempre conseguira ser la unica respuesta a un problema oclusal y estetico como el relatado, de ahí que un diagnóstico adecuado, empleando todos los 
metodos existentes, siempre será la herramienta mas adecuada para alcanzar el exito. Para una oclusión ideal es importante alcanzar en la pocición de máxima intercuspidación la mayor cantindad de puntos de contacto en todo los dientes, lo que fue verificado en este caso clinico con la ayuda de papel articular, se comprobo que se tenia puntos de contacto en todos los dientes, brindandonos una oclusión armónica ${ }^{(16)}$

El éxito del tratamiento se fundamentó en mantener la estabilidad de los tejidos periodontales, recuperación de la función masticatoria y dimensión vertical; y la obtención de un resultado estético aceptable y funcional de la paciente

Los tratamientos de rehabilitación ejecutados permitieron devolver la estética y la función del sistema masticatorio en una paciente con atrición dental anterior y pérdida de contención posterior. el éxito de cualquier tratamiento de rehabilitación oral está determinado por una buena anamnesis y un plan de tratamiento previamente elaborado, las acciones ejecutadas permitieron establecer la estética en el sector anterior mediante prótesis fija.

\section{Contribuciones de autoría:}

MAM, OGC, AAV, EV y ACG participaron en el diagnóstico, planificacion y tratamiento del caso., redacción y revisión crítica del artículo. los autores aprobaron la la versión final del artículo

Fuente de financiamiento: Autofinanciado.

Conflictos de interés: Los autores declararon no tener conflicto de interés.

\section{REFERENCIAS BIBLIOGRÁFICAS}

1. Alzoubi F, Bedrossian E, Wong A, Ferrell D, Park $C$, Indresano $\mathrm{T}$. Outcomes Assessment of Treating Completely Edentulous Patients with a Fixed Implant-Supported Profile Prosthesis Utilizing a Graftless Approach. J Coll Physicians Surg Pak. 2017; 32(5):1080-1085.

2. Akhtar Q, Danyal S, Zareen S, Ahmed B, Maqsood M, Azad AA. Clinical Evaluation of Proximal Contact Points in Fixed Prostheses. J Coll Physicians Surg Pak. 2015; 25(9):702-4.

3. Chou JC, Thompson GA, Aggarwal HA, Bosio JA, Irelan JP. Effect of occlusal vertical dimension on lip positions at smile. J Prosthet Dent. 2014;112 (3):533-9.

4. Kizilova N, Geramy A, Romashov $\mathrm{Y}$. Biomechanical analysis of asymmetric mesio- distal molar positions loaded by a symmetric cervical headgear. Acta Bioeng Biomech. 2016; 18(4):97-106.

5. Reimann $Ł$, Żmudzki J, Dobrzański LA. Strength analysis of a three-unit dental bridge framework with the Finite Element Method. Acta Bioeng Biomech. 2015; 17(1):51-9.

6. Santos $F$, Branco A, Polido $M$, Serro AP, Figueiredo-Pina CG. Comparative study of the wear of the pair human teeth/Vita Enamic $\AA$ vs commonly used dental ceramics through chewing simulation. J Mech Behav Biomed Mater. 2018: 20 (88):251-260.

7. Sasaki K, Yamamoto T, Ikawa $T$, Shigeta $Y$, Shigemoto S, Ando E, et al. Pre-endodontic Post and Core Technique for Endodontic and Prosthodontic Treatment. J Contemp Dent Pract. 2018; 19(1):117-122.

8. Sarver DM. Interactions of hard tissues, soft tissues, and growth over time, and their impact on orthodontic diagnosis and treatment planning. Am J Orthod Dentofacial Orthop. 2015; 148(3):380-6.

9. Sutter BA. Incidence of headaches related to occlusion and bite force imbalance: a case study. Cranio. 2015; 23(1):1-5.

10. Tezulas E, Yildiz C, Evren B, Ozkan Y. Clinical procedures, designs, and survival rates of allceramic resin-bonded fixed dental prostheses in the anterior region: A systematic review. J Esthet Restor Dent. 2018: 16

11. Ülkü SZ, Acun Kaya F, Uysal E, Gulsun B. Clinical Evaluation of Complications in ImplantSupported Dentures: A 4-Year Retrospective Study. Med Sci Monit. 2017; 23(1):6137-6143.

12. Ward DH. Proportional Smile Design: Using the Recurring Esthetic Dental Proportion to Correlate the Widths and Lengths of the Maxillary Anterior Teeth with the Size of the Face. Dent Clin North Am. 2015; 59(3):623-38.

13. Nomura Suzy, Freitas Karina Maria Salvatore. Evaluation of the attractiveness of different gingival zeniths in smile esthetics. Dental Press J. Orthod. 2018; 23(5): 47-57.

14. Tallarico M, Caneva M, Baldini N. Patientcentered rehabilitation of single, partial, and complete edentulism with cemented- or screwretained fixed dental prosthesis: The First Osstem Advanced Dental Implant Research and Education Center Consensus Conference 2017. Eur J Dent. 2018; 12(4):617-626.

15. Hüttig $F$, Keitel J, Prutscher A, Spintzyk S, Klink A. Fixed Dental Prostheses and Single-Tooth Crowns Based on Ceria-Stabilized Tetragonal Zirconia/Alumina Nanocomposite Frameworks: Outcome After 2 Years in a Clinical Trial. Int $\mathrm{J}$ Prosthodont. 2017; 30(5):461-464.

16. Nicolaisen M, Bahrami G, Schropp L, Isidor F. Functional and Esthetic Comparison of MetalCeramic and All- Ceramic Posterior Three-Unit Fixed Dental Prostheses. Int J Prosthodont. 2016; 29(5):473-81 
Marta Acosta Molina marthaacosta@hotmail.com

Osmani Guevara Cabrera osmaniguevara2323@hotmail.com

Ana Armas Vega anadelcarmen4@hotmail.com

Esteban Vizcaíno estebanvizcaino2123@hotmail.com

Alexander Cruz Gallegos alexandercruz1993@hotmail.com
(1) https://orcid.org/0000-0001-6048-5851

(b) https://orcid.org/0000-0002-1875-1254

(b) https://orcid.org/0000-003-0276-0356

(b) https://orcid.org/ 0000-0002-3477-6953

(b) https://orcid.org/0000-0001-9115-4512 